\title{
Massive Transfusion and Massive Transfusion Protocols. Where We Are and Where We Need to Go.
}

\section{Raul Coimbra}

Division of Trauma, Surgical Critical Care, Burns, and Acute Care Surgery, University of California San Diego, USA

Corresponding author:

Raul Coimbra, MD, PhD, FACS

The Monroe E. Trout Professor of Surgery

Surgeon-in-Chief UCSD Health System Hillcrest Campus

Executive Vice-Chairman Department of Surgery

Chief Division of Trauma, Surgical Critical Care, Burns, and Acute Care Surgery University of California San Diego Health Sciences

E-mail: rcoimbra@ucsd.edu
Bleeding is the most frequent cause of death following injury. Bleeding may be external, internal, acute, indolent, controllable, massive, organ-specific or diffuse due to coagulopathy, hypothermia and acidosis, the lethal triad.

The definition of Massive Transfusion, although intuitive and related to massive or significant continued hemorrhage, has varied in several studies, creating confusion and difficulties for data comparison between studies and scientific interpretation. The most commonly accepted definition of massive transfusion is that of greater than 10 units of packed red blood cells (PRNC's) transfused in a $24 \mathrm{~h}$ period. Although not ideal, this definition is easy to apply and allows some uniformity if applied consistently across the board.

It turns out that only $3 \%-8 \%$ of all injured patients admitted to trauma centers will require massive transfusion and $24 \%$ of those presenting in shock will be massively transfused. It becomes apparent that being prepared with process in place to quickly and effectively make blood available to injured patients is of utmost importance. The real issue is how to identify early on those patients who will go on to require blood transfusion. Classic factors related to massive blood loss include, but are not limited to multicavitary or transpelvic trauma, large hemoperitoneum identified by FAST, patients admitted with hypotension (Systolic Blood pressure $<90 \mathrm{mmHg}$ ) and tachycardia (Heart Rate $>120$ ), Significant base deficit in the trauma bay or emergency department, and those presenting with elevated INR due to injury severity or use of anticoagulants such as Coumadin and other new anticoagulants.

The initial management and resuscitation of the massively 
bleeding trauma patient has evolved in the last decade. A significant paradigm shift has occurred and the focus has gone from fluid resuscitation (primarily with crystalloids) to bleeding control first accompanied by early transfusion of blood and blood products to overcome the effects of injury and blood lossinduced coagulopathy. Two new concepts emerged from this paradigm shift: 1) Permissive Hypotension and 2) Damage Control Resuscitation. Data from the US military obtained during the last decade of military conflict in the Middle East demonstrated that using less crystalloids, focusing on bleeding control and using whole blood (if available) or a higher ratio of Fresh Frozen Plasma (FFP) to PRBC and Platelets (1:1:1) improves outcomes. Several studies have concluded that the principles of resuscitation proposed in the military studies are also applicable to the civilian environment. Others have also evaluated the use of fibrinogen and cryoprecipitate as adjuncts to PRBC, FFP, and Platelets, concluding that blood loss, transfusion requirements, and mortality are decreased when these products are used. In addition, other adjuncts such as Tranexemic Acid, Prothrombin Complex, and Activated Factor VIIa have been shown to affects outcomes when used appropriately, at the right time, and in the right patient.

Massive Transfusion Protocols exist to meet the requirements of Damage Control Resuscitation. The strategy includes starting blood early as the first line of fluid replacement, delivering $\mathrm{O}$ negative blood first, followed by type specific as soon as possible, and maintaining a steady supply at a PRBC;FFP:PLT ratio as close to 1:1:1, until it is no longer necessary. The goals are to treat traumatic coagulopathy at presentation, prevent dilutional coagulopathy, and prevent complications associated with massive hemorrhage/transfusion. Using classic transfu- sion triggers such as $6 \mathrm{U}$ to $10 \mathrm{U}$ of $\mathrm{PRBC}$ ) to initiate FFP and Platelet transfusion will only delay early correction of coagulopathy.

Any institution treating injured patients should have a an "Institutional Massive Transfusion Protocol" in place. Several studies comparing periods of time pre and post the implementation of a massive transfusion protocol have consistently demonstrated an increased ratio of PRBC:FFP, earlier initiation of transfusion, decreased crystalloid use, and decreased mortality at $24 \mathrm{~h}$ and $30 \mathrm{~d}$. Other have also shown decreased overall usage of blood products, decreased cost and decreased morbidity such as pneumonia, acute kidney injury, abdominal compartment syndrome, ARDS, sepsis, septic shock, and multiple organ failure. Whether massive transfusion protocols should be "laboratory-directed" or protocol driven is still a matter of discussion. The turnaround times for classic coagulation tests varies from 30-60 minutes and their accuracy is questionable in hypothermic patients. It seems that if one decides to use a laboratory-directed massive transfusion protocol it must be based on Thromboelastogram (TEG/ROTEM) findings, which have shown to predict and trigger the initiation of a massive transfusion protocol and are also applicable for ongoing goal-directed blood component therapy.

In summary, massive transfusion protocols improve immediate availability of blood products, likely decrease mortality when used effectively and timely, and may decrease costs and blood product utilization. A massive transfusion protocol should be tailored to the types of patients treated at the institution and should be embraced not only by the trauma surgeons, but also by transfusion medicine, emergency medicine, and other surgical specialties. Consistency in timely blood product delivery saves lives. 